\title{
Design of a Power-Efficient Low Complexity Non Maximally Coefficient Symmetry Multi Rate Filter Bank for Wideband Channelization
}

\author{
Kirti Samir Vaidya ${ }^{1}$, C. G. Dethe ${ }^{2}$, S. G. Akojwar ${ }^{3}$ \\ ${ }^{1}$ Research Scholar in Rajiv Gandhi college of Engineering and research Gondwana, in Department of Electronics and \\ Telecommunication Engineering, Maharashtra, \\ Email Id: kirtismir87n@gmail.com \\ ${ }^{2}$ Director ,UGC Human resource department, \\ Rashtra Sant Tukdoji Maharaj University, \\ Nagpur. Email Id: dethecg605@gmail.com \\ ${ }^{3}$ Principal in Government College of Engineering, Chandrapur, Maharashtra, \\ Email Id:akojwaar90sg@gmail.com
}

Received: March 1, 2021. Revised: July 21, 2021. Accepted: August 4, 2021. Published: August 6, 2021.

I. INTRODUCTION

\begin{abstract}
A solution for existing and upcoming wireless communication standards is a software-defined radio (SDR) that extracts the desired radio channel. Channelizer is supposed to be the computationally complex part of SDR. In multi-standard wireless communication, the Software Radio Channelizer is often used to extract individual channels from a wideband input signal. Despite the effective channelizer design that reduces computing complexity, delay and power consumption remain a problem. Thus, to promote the effectiveness of the channelizer, we have provided the Non-Maximally Coefficient Symmetry Multirate Filter Bank. In this paper, to improve the hardware efficiency and functionality of the proposed schemes, we propose a polyphase decomposition and coefficient symmetry incorporated into the NonMaximally Coefficient Symmetry Multirate Filter Bank. For sharp wideband channelizers, the proposed methods are suitable. Furthermore, polyphase decomposition filter and coefficient symmetry is incorporated into the Non-Maximally Coefficient Symmetry Multirate Filter Bank to improve the hardware efficiency, power efficient, flexibility, reduce hardware size and functionality of the proposed methods. To prove the complexity enhancement of the proposed system, the design to be the communication standard for complexity comparison.
\end{abstract}

Keywords-Software Defined Radio, Wireless Communication, polyphase decomposition, NonMaximally Polyphase Decimated Filter, Frequency Response Masking, Coefficient Symmetry

\begin{abstract}
COMMUNICATION developments include the IoT, cooperative communication, cognitive radio, wireless technology has rapidly arisen, representing signal processing requirements. The high sampling frequencies and data rates describe the signal processing operations due to the higher number of operations each second in wideband systems [1]. The usage
\end{abstract}

of broadband systems is resulting the complexity of the hardware [2], which is gradually improved during the transmission of high data rate. The high throughput and less power consumption describe the new outlook in rapidly growing communication technology [3].

Increasing the demand for effective communication systems is a prominent factor that is endorsing the development of the SDR. Communication serves with high frequency a high range of industry verticals ranging from too few consumer electronic devices to telecommunication infrastructure equipment and too big military devices. In SDR-based multi-standard radios, the channelizer usually identifies specific radio channels from the wideband input signal [4]. Channel filtering, sampling rate conversion, and digital down-conversion are generally included in Software radio channelizers. In a cognitive radio system, based radio software was an essential component. Modern SDR receivers can accept many channels with various communication standards is of importance on provided bandwidth. The Multirate digital filter bank was used in the radio channel application to eliminate the wideband given signal's wideband channels $[5,6]$.

To extract multiple narrowband channels from the received wideband signal, digital filter banks in the SDR receiver [7] usually employ a channelizer. There are many papers which use FRM technique for sharp filter implementation in SDR channelizers. This arbitrary interpolator-based FRM 
channelizer can channelize wideband signal into narrow sub-bands, but the hardware complexity of this structure is also very high. The polyphase filter bank system, which is constituted by the polyphase components of the analysis and synthesis of prototype filters, offers good power efficiency. Based on the decimation factor used, the polyphase filter banks can be classified as maximally and non-maximally decimated. In maximally decimated polyphase filter banks, there may be aliasing between adjacent bands of each polyphase path.

The filter bank channelization was mounted instantly after ADC, which should perform at an increased sampling rate in the digital front-end of the receiver. If the channelizer has most computationally intensive components of a wideband receiver system, this block absorb the power and make it computer-intensive. Therefore, efficient architectures of low complexity are necessary for the channelizer to be incorporated.

A filter bank scheme is one of the successful solutions to fulfill these criteria. The hardware and computational complexity of the channelizer based on filter banks is, preferring efficient wideband channelization. These days, the spectrum has become restricted, and sharp filters are necessary within wideband channelizer to achieve proper spectrum usage. Sharp filters frequently help in minimizing interaction with the adjacent channel [8]. Understanding sharp transformation width reactions on filter bank-depend system were too costly in terms of monitoring and budget.

In the study papers, several methods of designing the filter bank are usable. Uniform filter banks and here channels were identical bandwidths [9], non-uniform filter banks where channels are unequal bandwidths [10]. Also, reconfigurable FB where channels are variable bandwidths FRM is the commonly utilized method for generating low complexity and sharp transition distance channels.

\section{RELATED WORK}

In [11] developed the compact, system-level modelling method in the design of a digital channelizer. This approach applied effective MDP to design optimization for reconfigurable channelization processing. The technique was used to produce the runtime reconfiguration for a timevarying environment. But, high computational complexity, delay, wrong tuning is possible utilizing this approach. To promote the low complexity and reconfigurability of filter banks in SDR receivers, the Fourier transform (DFT) depend on FB. The implementations fulfil stringent criteria of Software Defined Radio receivers provided in [12]. Filter bank depends upon FRM will be utilized as an adequate replacement for a per-channel method to be less complex, and the FB depends on coefficient decimation method is being used as the substitute to FB with less complexity. MFIR filter was utilized as the prototype filter to achieve a sharp transition width with less hardware complexity. A proposed structure used in an SDR channel for understanding multiple channels was related to reconfiguration, hardware efficiency, and state-of-the-art structures. Discrete Fourier altered filter for the design of non-uniform near-ideal reconstruction to implement reconfigurable software-defined wireless radio channelling systems include very minimum hardware complexity. Anon-uniform modified Discrete Fourier transform filter bank was acquired by combining adjacent channels of the uniform modified Discrete Fourier transform. In this channelized design, complexity, area, power are the critical problems. Uniform channelization coefficient decimation based on the filter bank, utilizing the FIR filter [13] for SDR channelization. By coefficient decimation, the low pass, high pass, bandpass responses were provided. The maximum resampling ratios require low-pass digital filters include a very narrow transition band leads to increased calculation complexity and makes filter design difficult. Thus, the Frequency Response Masking process breaks filter with the narrow transition band to another set of filters with decreased design requirements [14]. It reduces the quantity of non-zero coefficients, and an effect, the computational complexity of the resampler is diminished by using the FRM filter. The leading decimated filter bank should not vary from multiple channels that are not flexible for the low-complexity channelizer.

To reduce the high computational complexity of the narrow transition bandwidth filter bank, In [15] provided a low complexity channelizer depending on the frequencyresponse masking (FRM). The half-band filter was utilized to build a prototype filter of present less-complexity channelize depend on Frequency Response Masking. Twotier and interleaving schemes operating NMDFB explained in paper [16] were the best methods in channelizer design. Although it has some drawbacks includes reduced flexibility and reduced reconfigurability in the production of the high no of stringent bandwidth and too sharp transition width responses. The benefit is that it requires limited resources and, while the delay is a significant issue, has less difficulty.

\section{METHODOLOGY}

\section{A. Frequency Response Masking Techniques}

FRM method [17] was acceptable for designing digital filters, including sharp transition bands. A sharp transition bandwidth is achieved by combining broad transition band filters, typically known as masking filter and model filter. The model filter was upsampled to develop a periodic model filter that, as a result of upsampling, compresses the filter's frequency response to obtain an optimal frequency band with a sharp transition band. Also, due to the upsampling, there were extra frequency components in the spectrum. These spectral filters are eliminated by cascading the model filter with masking filters.

The cascading of non-periodic filters with periodic filters to achieve sharp transition band the Frequency Response Masking structure method in Fig1 allows arbitrarybandpass FIR filters with low hardware complexity to be implemented most filter coefficients were zero. Utilizing the FRM method for designing filter with some multipliers [18] could decrease the hardware complexity of FIR filters. The sparse nature of Frequency Response Masking filter coefficients enables the filter less hardware complex for 
high-resolution uses. Provided the prototype symmetrical impulse response linear phase less-pass filter $S_{a}(z)$ acalled as prototype filter or Model filter for $\operatorname{order} L_{a}$, the complementary filter $S_{c}(z)$ couldrepresented as, $S_{c}(z)=z^{-\left(L_{a}-1\right) / 2-S_{a}(z)}$

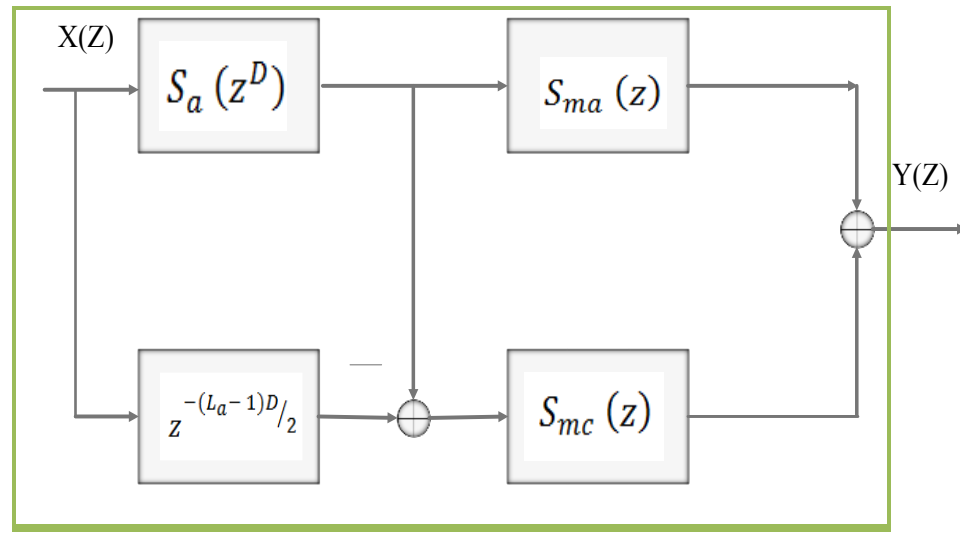

Fig 1.FRM structure

The delay of the model filter, such as $S_{a}(z)$ and $S_{c}(z)$ is removed successfully by replacing the $\mathrm{D}$ delays outcoming in interpolated linear-phase FIR filters $[19] S_{a}\left(z^{D}\right)$ and $S_{c}\left(z^{D}\right)$. The transition width of these IFIR filter is $\frac{1}{D}$ times that of $S_{a}(z)$. As given in Fig. Two, Both masking filters $S_{m a}(z) S_{m c}(z)$ are cascade to $S_{a}\left(z^{D}\right)$ and $S_{c}\left(z^{D}\right)$, correspondingly, as given in Fig. Two, in FRM techniques. [27] provided a transfer function for filtering,

$S(z)=S_{a}\left(z^{D}\right) S_{m a}(z)+S_{c}\left(z^{D}\right) S_{m c}(z)$

Equation 3 can be expressed using (1) and (2) as follow,

$S(z)=S_{a}\left(z^{D}\right) S_{m a}(z)+z^{-\left(\frac{L_{a-1}}{2}\right) D}-S_{a}\left(z^{D}\right) S_{m c}(z)$

In FRM, the prototype model filter $S(z)$ was interpolated by the various values that is $2,4,8,16$, and 32 or interpolated factor and by cascading the interpolated sub-filter include $S(z)$ or $S_{c}(z)$ Might produce needed bandpass filters. Therefore, any sharp transition-band FIR filter could be implemented with less complexity by choosing stopband and passband edges of modal and masking filters. Modified
Frequency Response Masking using IFIR was presented to be following in Fig.4,

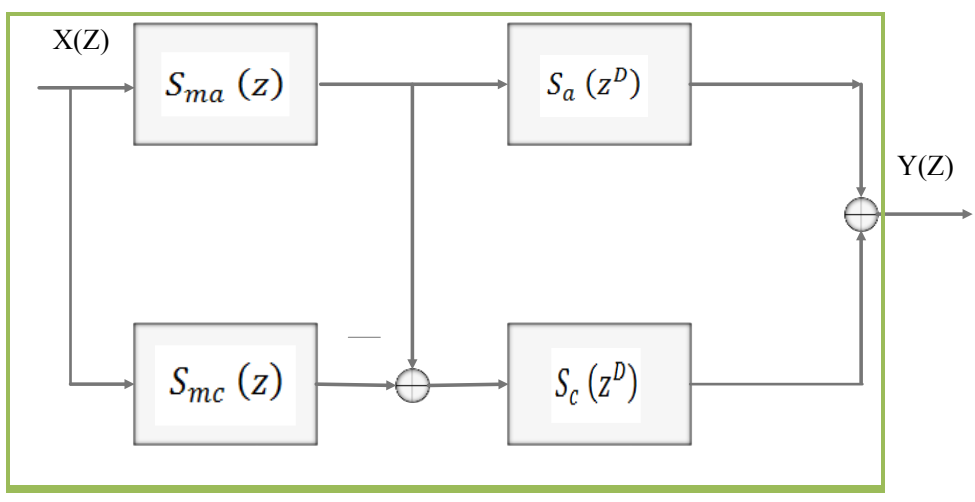

Fig 2.Modified FRM

Increased data rates and sampling frequency in many operations per second are needed when using the wideband system in signal processing. The multi-rate decimation and interpolation operations implemented decompose any system function $\mathrm{H}(\mathrm{z})$ into its polyphase representation [20].

\section{B. Proposed Polyphase Decomposition}

Polyphase decomposition of a filter is the splitting of the filter into various sub-filters. This technique results in computationally efficient implementations of decimation and interpolation filters [30]. The transfer function of a digital filter can be written as,

$$
\begin{aligned}
& H(z)=\sum_{n=-\infty}^{\infty} h(n) z^{-n} \\
& H(z)=\sum_{k=0}^{M-1} z^{-k} E_{k}\left(z^{M}\right) \\
& E_{k}(z)=\sum_{n=-\infty}^{\infty} e_{k}(n) z^{-n}, 0 \leq k \leq M-1 \\
& e_{k}(n) \Delta h(M n+k)
\end{aligned}
$$

Using $M$-level polyphase representation $H(z)$ can be written as, [30] 


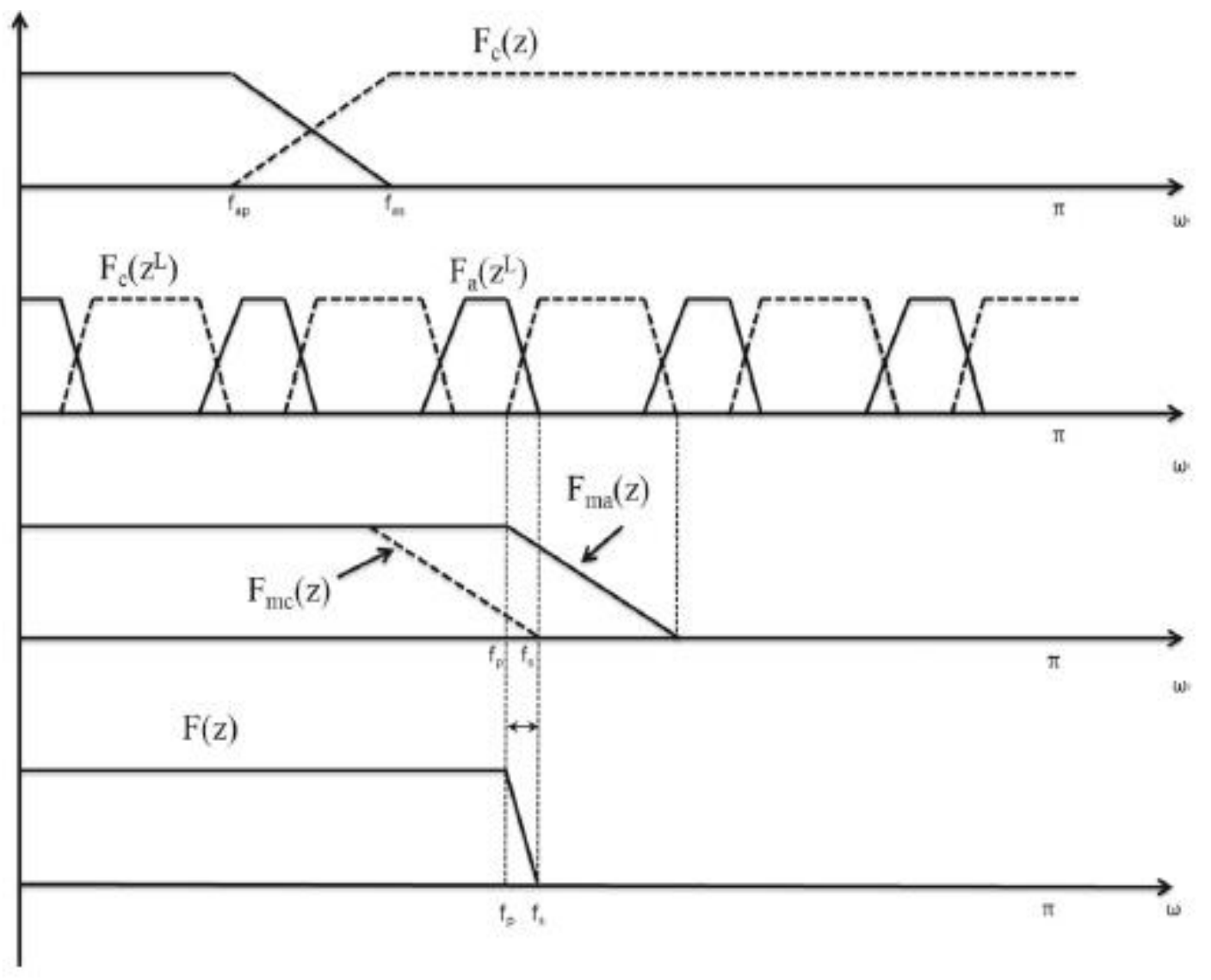

Fig. 3 Illustration of FRM

$E k(\mathrm{zm})$ represents the polyphase components of $H(z)$ and Eq. 5 represents the $M$ level Type 1 polyphase representation. A different polyphase representation known as Type-2 representation of $H(z)$ is given by [30],

$$
\begin{aligned}
& H(z)=\sum_{k=0}^{M-1} z^{M-1-k} R_{k}\left(z^{M}\right) \\
& R_{k}(z)=E_{M-1-k}(z), \quad 0 \leq k \leq M-1
\end{aligned}
$$

$R k(z)$ represents the Type-2 polyphase components of $H(z)$.

The polyphase implementation of a filter requires less number of multiplications and additions per unit time than the conventional implementation. For a filter of order $N$, the $M$ level polyphase implementation requires only $(N+$ 1) $/ M$ multiplications per unit time and $N / M$ additions per unit time. Also, the input signal is processed in parallel at a reduced sampling rate. Thus, the polyphase decomposition offers a power-efficient implementation of filters.

\section{Proposed Non-Maximally Coefficient Symmetry Multi- Rate Filter Bank}

In this section, the Non-Maximally Coefficient Symmetry Multirate Filter Bank is proposed to reduce the delay and hardware complexity for a wideband channelizer. The new design is based on the Reconfigurable FRM framework in which interpolated linear phase FIR filters were utilized to understand the non-maximally polyphase decimated filter. Also, different masking responses were realized utilizing NMDFB in this structure. The advantage of using architecture is the single analysis filter bank could be used to recognize responses of two masking filters. The selection of the channel is applied to the inputs of the appropriate synthesis bank. Thus, the entire masking procedure can be understood by utilizing the single Analysis FB and both synthesis filter banks. In instances where the complexity of specification played a significant role, the architecture can be chosen based on the modal filter order, and prototype filter analysis was built, as shown in Fig.3.Also, it is possible to integrate hardware-level optimization into the updated FRM approaches employing coefficient symmetry in NMDFB polyphase filters to improve the efficiency of hardware and flexibility of presented schemes. 


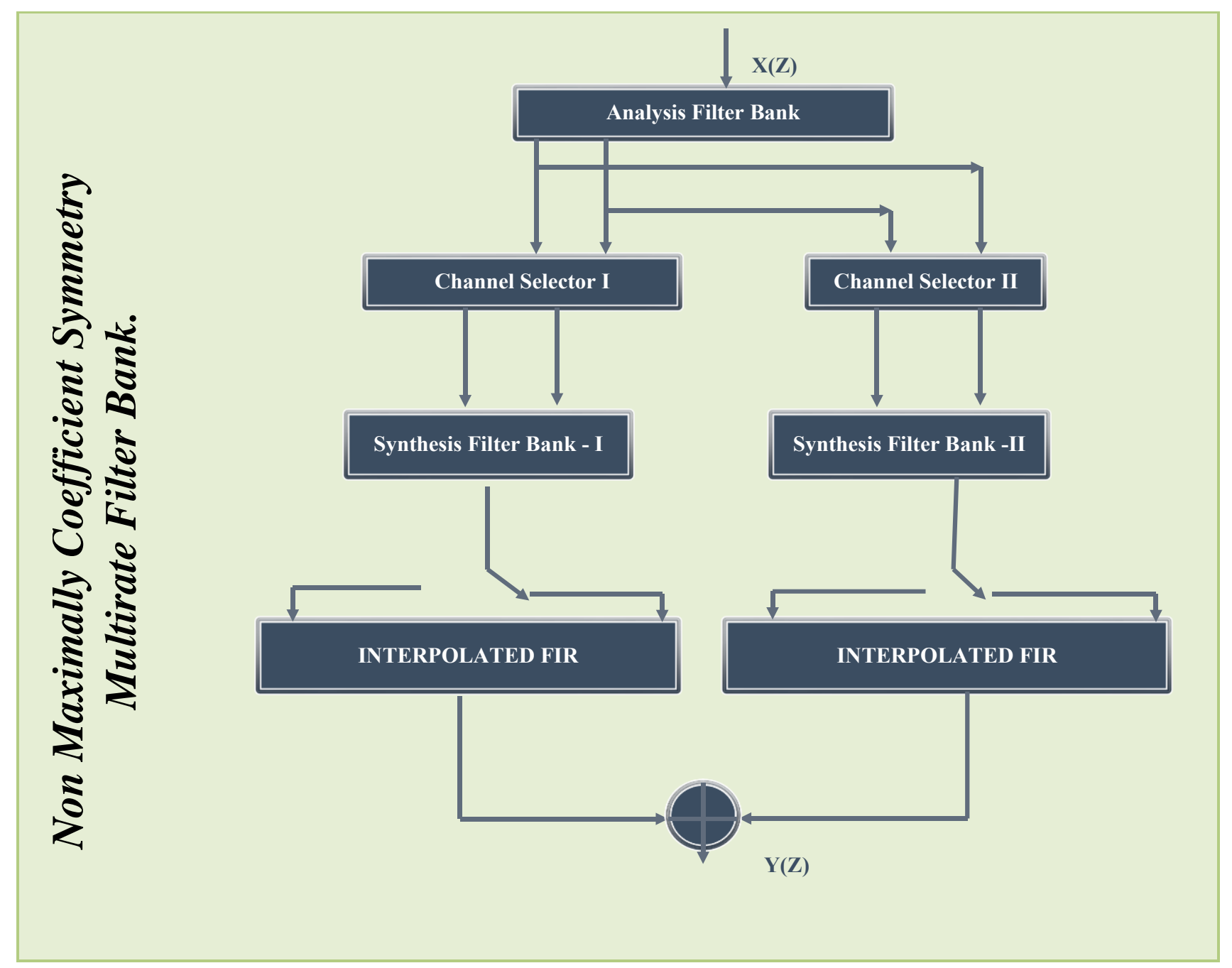

Fig 4.Proposed Non-Maximally Polyphase Decimated Filter

\section{Non-Maximally Decimated Polyphase Filter Bank}

Polyphase was the way for doing a sampling-rate conversion, which leads to high efficient implementations. Using this implementation of polyphase decompose, the polyphase filter was designed [21]. A polyphase bank consists of an analysis stage, and a synthesis stage of prototype filters offers good power efficiency. Each stage consists of a set of filters in parallel. Each step consists of a group of filters in parallel. Depend on the decimation factor utilized, polyphase filter banks could be categorized as nonmaximally and maximally decimated. There might be aliasing among adjacent bands of every polyphase path in maximally decimated polyphase filter banks. This limits their utilize of large-spectral-resolution uses. NMDFB [22] faces the aliasing issue by raising the channel spacing. Therefore the polyphase NMDFB system was being used in work.

The changed structure where, decimated factor $<\mathrm{M}$ channel, of the single filter could be designed to satisfy perfect construction properties and process any arbitrarily situated frequency without folding noise. Generally, in
NMDFBs, $D$ is taken as $M / 2$. Thus, the outcome sampling frequency was twice that is it becomes $2 * \frac{\text { sampling rate }}{M}$ instead of $\frac{\text { sampling rate }}{M}$ as a maximally decimated filter bank. Polyphase Analysis achieves it, and Synthesis Filter Bank for Non-maximally destroyed filter banks as follow, M-path polyphase decomposition of prototype filter was provided as [28]

$H(z)=\sum_{k=0}^{M-1} z^{-k e^{\frac{j 2 \pi m k}{M}}} C_{k}\left(z^{\frac{M}{D}}\right), 0 \leq m \leq M-1$

where, $k=0,1,2, \ldots, M-1$, D was a decimation factor, M-parallel paths, $m$ was a channel number and $C_{k}\left(z^{\frac{M}{D}}\right)$ werekth polyphase components achieved by polyphase decomposition of $H(z)$ where an M-path delayed down-sampled commutator accepts $\mathrm{M} / 2$ given data sequences at the sampling frequency of $\mathrm{fs}$ and produce result samples at the frequency of $2 * \frac{\text { sampling rate }}{M}$. Hence, the polyphase representation of analysis and synthesis filter will be modified as, 
$A_{m}^{p}(z)=\sum_{k=0}^{M-1} z^{-k} e^{\frac{j 2 \pi m k}{M}} C_{k}\left(z^{\frac{M}{D}}\right), 0 \leq m \leq M-$

1

$S_{m}^{p}(z)=\sum_{k=0}^{M-1} Z^{-k} e^{\frac{j 2 \pi m k}{M}} C_{k}\left(z^{\frac{M}{D}}\right), 0 \leq m \leq M-1$

$A_{m}^{p}(z)$ and $S_{m}^{p}(z)$ can also express as given, [26] based on Aliasing cancellation condition, Distortionless condition

$A_{m}^{p}(z)=A\left(z W_{M}\right)$

$S_{m}^{p}(z)=S\left(z W_{M}\right)$

Condition for eliminating the folding noise or aliasing $A^{p}\left(z W_{D}^{d}\right) S^{p}(z)=0 ; \forall d=1,2, \ldots D-1$

These equations impose the condition in responses of $A_{m}^{p}(z)$ and $S_{m}^{p}(z)$ such that $S_{m}^{p}(z)$ and shifted version of $A_{m}^{p}(z)$ may have reduced overlap to make reduced aliasing. By properly setting the passband and the stopband frequencies of the prototype filters and choosing filters with a high stopband performance, aliasing energy can be reduced to a considerably low value. Therefore, the NMDFB is recommended over MDFB for wideband SDR channelizer designs.

\section{Coefficient Symmetry in Non-Maximally Coefficient Symmetry Multi-Rate Filter Bank}

Reducing the complexity of filter banks has become a concern research topic in the final decades. For that, symmetric has been successfully used in the design of

$$
\begin{aligned}
\mathrm{A}(\mathrm{z}) \text { or } \mathrm{H}(\mathrm{z}) & \left.=\sum_{k=0}^{R-1} z^{-k}\left(C_{k, 1}^{R}\left(z^{M}\right)+z^{-M\left[\frac{N}{M}\right] / 2} C_{k^{\prime}, 1^{\prime}}^{R}\left(z^{M}\right)\right)\right) \\
& \left.+\sum_{k=R}^{\left[\frac{M+R}{2}-1\right]} z^{-k} C_{k, 1}^{S 1}\left(z^{M}\right)+z^{-M\left[\frac{N}{M}\right] / 2} C_{k^{\prime \prime}, 1^{\prime}}^{S 1}\left(z^{M}\right)\right) \\
& \left.+\sum_{k=\frac{M+R}{2}}^{M-1} z^{-k} C_{k, 1}^{S 1}\left(z^{M}\right)+z^{-M\left[\frac{N}{M}\right] / 1} C_{k^{\prime \prime}, 1^{\prime}}^{S 2},\left(z^{M}\right)\right)
\end{aligned}
$$

where $\mathrm{M}$ represents the number of parallel-connected sub filters, $\mathrm{N}$ denotes order of the filter, $N \backslash M$ indicates the subfilters order of the polyphase structure, $R=N-$ $M\left(\left\lceil\frac{N}{M}\right\rceil\right)+\mathrm{M}$ and $\mathrm{k}, k^{\prime}, k^{\prime \prime}$ are complementary sub filters. digital filter banks that required optimization. A better response characteristic is obtained and continuously minimizes the complexity of filter banks [23]. In this manner, we have presented the symmetric coefficient approach for modified FRM techniques using non maximally polyphase filters to decrease the use for multipliers in FB. We have deduced transfer function expressions to utilize the coefficient symmetry of modified FRM techniques using non maximally polyphase filters. It was shown that the presented structures could decrease multiplication complexity by a factor of 2 over the filter coefficient of sub-filters. The proposed filter's total transfer unction for every arbitrary positive integer values for $N$ and Mutilizing coefficient symmetry is derived under the following 2 instances,

a) While sub filters order of filter structure was even $(N \backslash M)$,

b) While sub filters order of filter structure was $\operatorname{odd}(N \backslash M)$,

\section{Decimator}

\section{When sub filters order of filter structure was even,}

In this condition, the transfer function of modified FRM techniques using non maximally polyphase filters using the equation $(A(z))$ is could expressed as below from [29],

$$
\begin{aligned}
\mathrm{A}(\mathrm{z}) & \left.=\sum_{k=0}^{\left[\frac{R}{2}-1\right]} z^{-k}\left(C_{k, 1}^{R 1}\left(z^{M}\right)+z^{-M\left[\frac{N}{M}+1\right]} / 2 C_{k^{\prime}, 1^{\prime}}^{R 1}\left(z^{M}\right)\right)\right) \\
& \left.+\sum_{k=\left[\frac{R}{2}\right]}^{[R-1]} z^{-k} C_{k, 1}^{S 2}\left(z^{M}\right)+z^{-M\left[\frac{N}{M}\right] / 2} C_{k^{\prime \prime}, 1^{\prime}}^{S 2}\left(z^{M}\right)\right)
\end{aligned}
$$

\section{When sub filters order of the filter structure is} odd,

In this condition, the transfer function of modified FRM techniques using non maximally polyphase filters using the equation (A ( $\mathrm{z})$ ) is could be expressed as below, 


$$
+\sum_{k=R}^{M-1} z^{-k} C_{k, 1}^{S}\left(z^{M}\right)+z^{-M\left[\frac{N}{M}-1\right]} / 2 C_{k^{\prime \prime}, 1}^{S}\left(z^{M}\right)
$$

\section{a. Numerical Analysis using Positive Integer Values of $N$ and $M$}

Considered, $\mathrm{R}=\mathrm{M}, \mathrm{M}=4$, and $\mathrm{N}=16$ into using this following equation even (10), the transfer function of the proposed filter structure could be represented as,

$\mathrm{A}(\mathrm{z})=\sum_{k=0}^{3} z^{-k}\left(\sum_{l=0}^{1} h_{4 l+k z^{-4 l}}\right)+z^{-8} \sum_{u=0}^{1} h_{4 u+k^{\prime} z^{-4(1-u)}}$ (18)

Using this equation, higher index coefficients in subfilters of the proposed filter are rejected and particular symmetric low index coefficients. Moreover, the multiplier unit is $\mathrm{N} \backslash 2$ instead of the conventional polyphase filter for multirate system. Considered $\mathrm{J}=\mathrm{M}=4$ in equation (17), higher index coefficients in subfilters of the proposed filter are rejected, and particular symmetric low index coefficients. Moreover, the multiplier unit is $N \backslash 2$. Furthermore, it can easily be recognized that the structure applicable for designing the proposed designing of multi-rate systems. Accordingly, we consider polyphase structure and downsampler (J) of $\mathrm{M}=\mathrm{J}=4$ was inserted at the end of filter result after selecting possible filter coefficients. (that is for demonstration, $\mathrm{c} 0$; $\mathrm{c} 1$; c2; c3; c4; c5; c6; c7; c8; c9; c10; c11; c12; c13; c14; and c15). After using noble identities, downsampler was inserted at filter result could be equally replaced by 4 down samplers at point B1, B2, B3, and B4. As a result of this, that structure will work as the decimator as shown in Fig. 4.

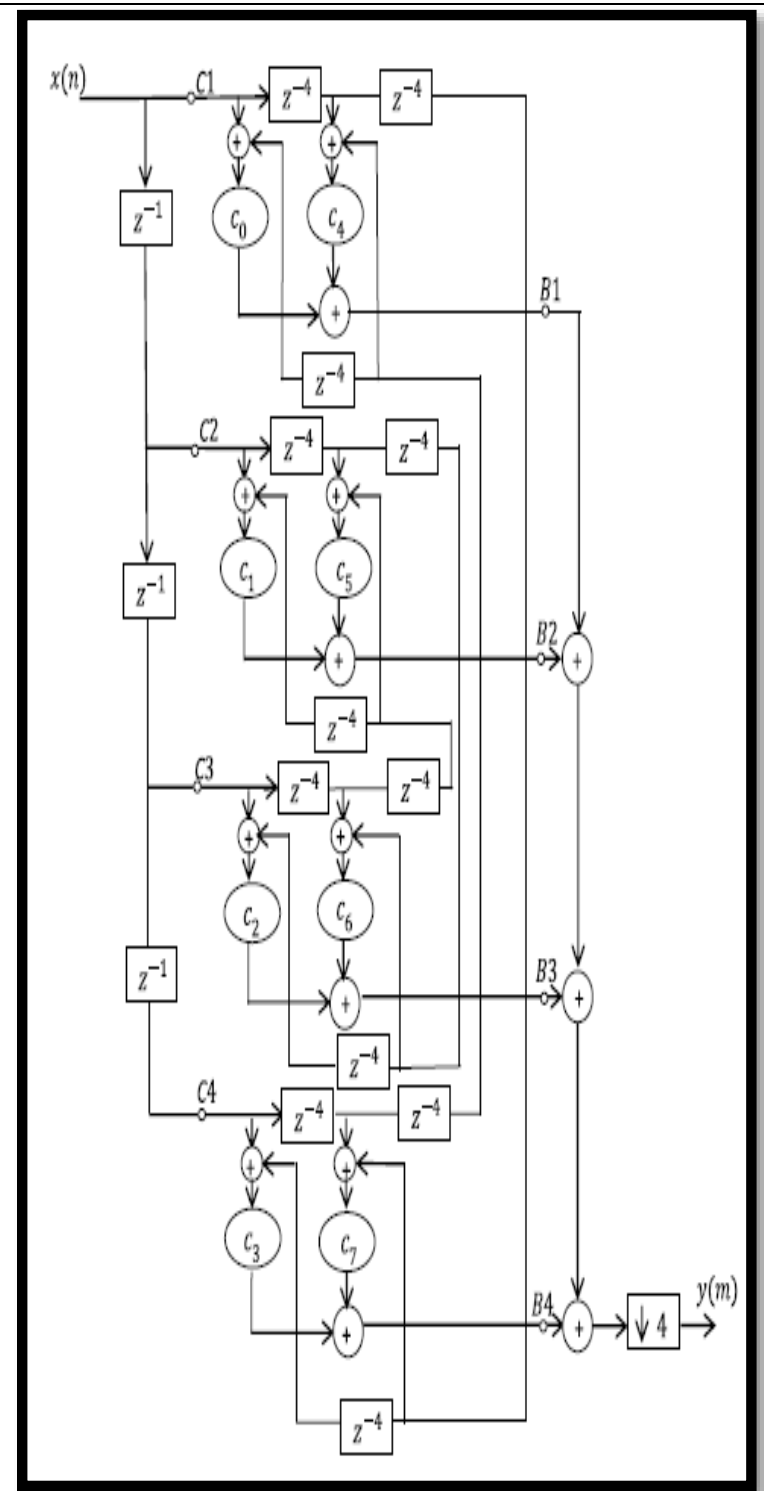

Fig 5.Conventional Polyphase Structure for Deciminator

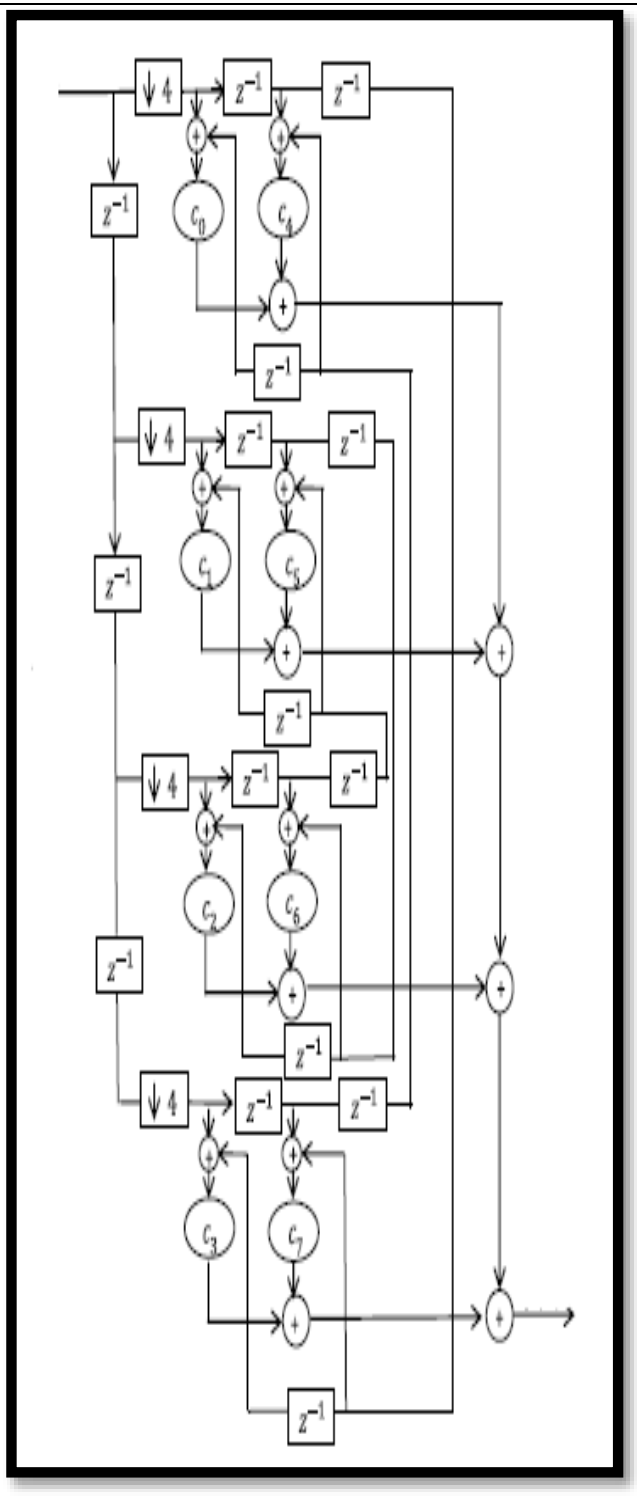

Fig 6.Modified Polyphase Structure for Deciminator

Although it was not able to perform filtering operation at a reduced sample rate. And also, As shown in Figure.5, 4 down sampler could equivalently be moved to point $\mathrm{C} 1$, 
$\mathrm{C} 2$, C3, and $\mathrm{C} 4$, replacing each delay element from $z^{-4}$ to $z^{-1}$. Although the desired decimator could be realized at a reduced sample rate. In modified FRM using the non maximally polyphase filter, two synthesis filter bank is used for wideband channelizer. In which the efficient transposed interpolator design is achieved using coefficient symmetry.

\section{- Transformed Interpolator}

While sub filters order of the filter structure is even,

$\mathrm{S}(\mathrm{z})=\sum_{k=0}^{R-1} z^{-k}\left(C_{k, 1}^{R}\left(z^{M}\right)+C_{k^{\prime}, 1}^{R}\left(z^{M}\right)\right)$

$$
\begin{aligned}
& \left.+\sum_{k=R}^{\left[\frac{M+R}{2}-1\right]} z^{-k} C_{k, 1}^{S 1}\left(z^{M}\right)+C_{k^{\prime \prime}, 1}^{S 1}\left(z^{M}\right)\right)
\end{aligned}
$$

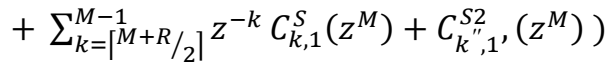

\section{When sub filters order of the filter structure is odd,}

$\left.\mathrm{S}(\mathrm{z})=\sum_{k=0}^{\left[\frac{R}{2}\right]-1} z^{-k}\left(C_{k, 1}^{R 1}\left(z^{M}\right)+C_{k^{\prime}, 1}^{R 1}\left(z^{M}\right)\right)\right)$

$$
\begin{gathered}
\left.+\sum_{k=\left\lceil\frac{R}{2}\right]}^{[R-1]} z^{-k} C_{k, 1}^{S 2}\left(z^{M}\right)+C_{k^{\prime \prime}, 1}^{S 2}\left(z^{M}\right)\right) \\
\left.+\sum_{k=R}^{M-1} z^{-k} C_{k, 1}^{S}\left(z^{M}\right)+C_{k^{\prime \prime}, 1}^{S}\left(z^{M}\right)\right)
\end{gathered}
$$

Considered, $\mathrm{J}=4, \mathrm{~N}=16$ and $\mathrm{R}=\mathrm{J}$ with every coefficient were positive $(+)$ into using this following equation even (19), the transfer function for proposed transform filter structure could be expressed as,

$$
\begin{aligned}
& \mathrm{S}(\mathrm{z})=\sum_{k=0}^{3} z^{-k}\left(\sum_{l=0}^{1} h_{4 l+k z^{-4 l}}\right)+ \\
& \sum_{u=0}^{1} h_{\left(4 u+k^{\prime} z^{-4(1-u)}\right) z^{-12+8 u}}
\end{aligned}
$$

Using this equation (21) on the conventional transposed polyphase FIR structure and proposed transposed architecture to evaluate the multiplier count. Thus, the $\mathrm{N} \backslash 2$

multiplier is required instead of N. Correspondingly, we consider that the conventional transposed polyphase structure and upsampler (L) of $\mathrm{M}=\mathrm{L}=4$ were inserted at the end of the filter result selecting possible filter coefficients. (that is for demonstration, $\mathrm{c} 0 ; \mathrm{c} 1 ; \mathrm{c} 2 ; \mathrm{c} 3 ; \mathrm{c} 4 ; \mathrm{c} 5 ; \mathrm{c} 6 ; \mathrm{c} 7 ; \mathrm{c} 8$; c9; c10; c11; c12; c13; c14; and c15). After using noble identities, the downsampler provided at filter result could equivalently replaced by 4 upsamplers at point B1, B2, B3, and B4. With this, that structure will work as the interpolator given in Figure. 6. Although it conducts filtering operation at increased sample rate. That is $4 f_{\text {in }}$. So that, As shown in Figure.7, By replacing every delay element from $z^{-4}$ to $z^{-1}$, the four upsamplers could equivalently be moved to point $\mathrm{C} 1, \mathrm{C} 2, \mathrm{C} 3$, and $\mathrm{C} 4$. Although, it carries out the filtering process at a higher sample rate. That is, $f_{\text {in }}$. And also, it was worth founded that filter structures (that is for even $(10,13)$ and odd $(11,14)$ ) were used to design multi-rate systems. 


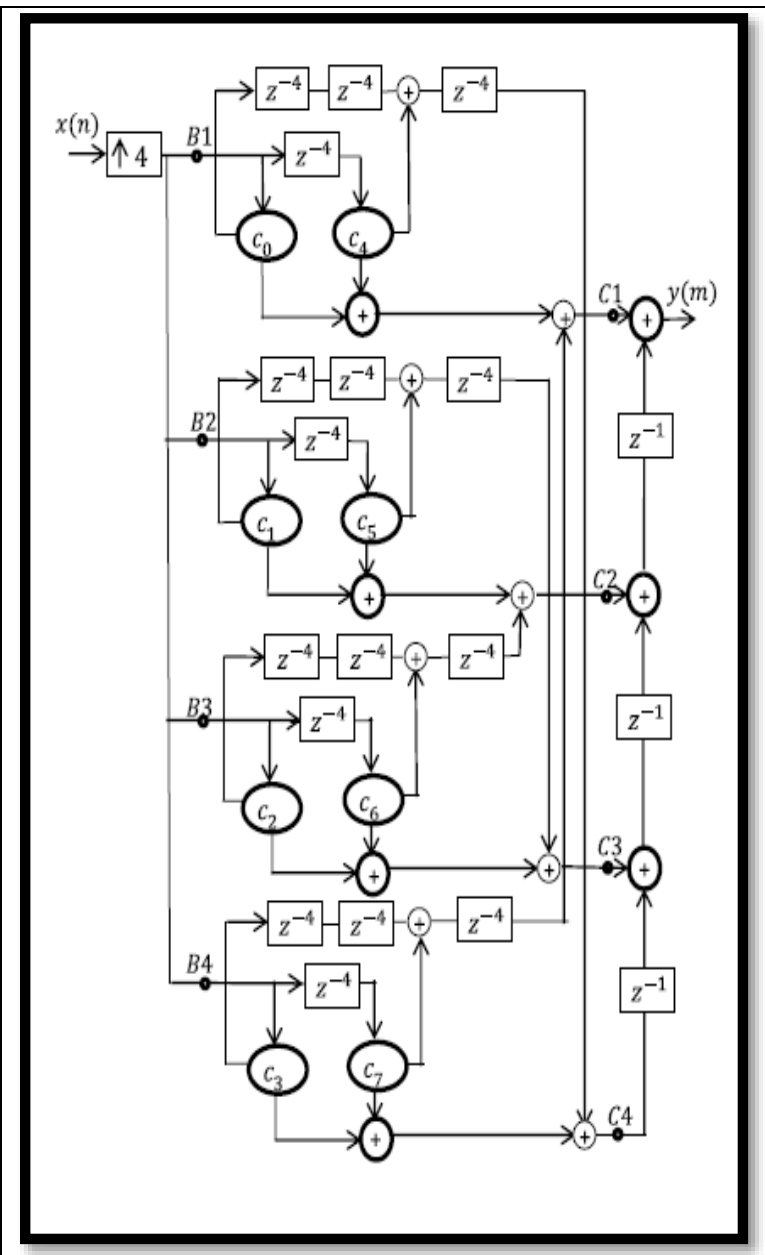

Fig 7. Conventional Transformed Polyphase Structure for Interpolator

\section{RESULT}

The performance of the reconfigurability, power efficiency and low complexity of a reconfigurable channel filter/filter banks of cell phone receivers is discussed in this section. Thus, the wideband channel of communication standards includes QPSK, FSK, and PSK used to analyze the proposed filters. Moreover, the intends to prove an advantage of the presented filter while compared to new techniques, communication standards. All the simulations are done in MATLAB.

We assumed the example of $\mathrm{N}=16$ and $\mathrm{J}=4$ to prove the feature of phase and magnitude to verify the proposed NonMaximally Coefficient Symmetry Multirate Filter Bank. As given in Fig3, For a valid relation, we plotted phase and magnitude characteristics. This can be noticed from Figs. 4 and 5 that we can obtain the particular magnitude by inserting the magnitudes of $\mathrm{k} \mathrm{D} 0 ; 1 ; 2 ; 3$ sub-filters with their corresponding phases. Besides, each sub-group filter's delay is static, leading to the linear phase of the proposed Multirate Filter Bank Non-Maximally Coefficient Symmetry. In successful decimator and interpolator structures, these linear-phase characteristics may provide the ability to review the signal on sub-filters beyond phase

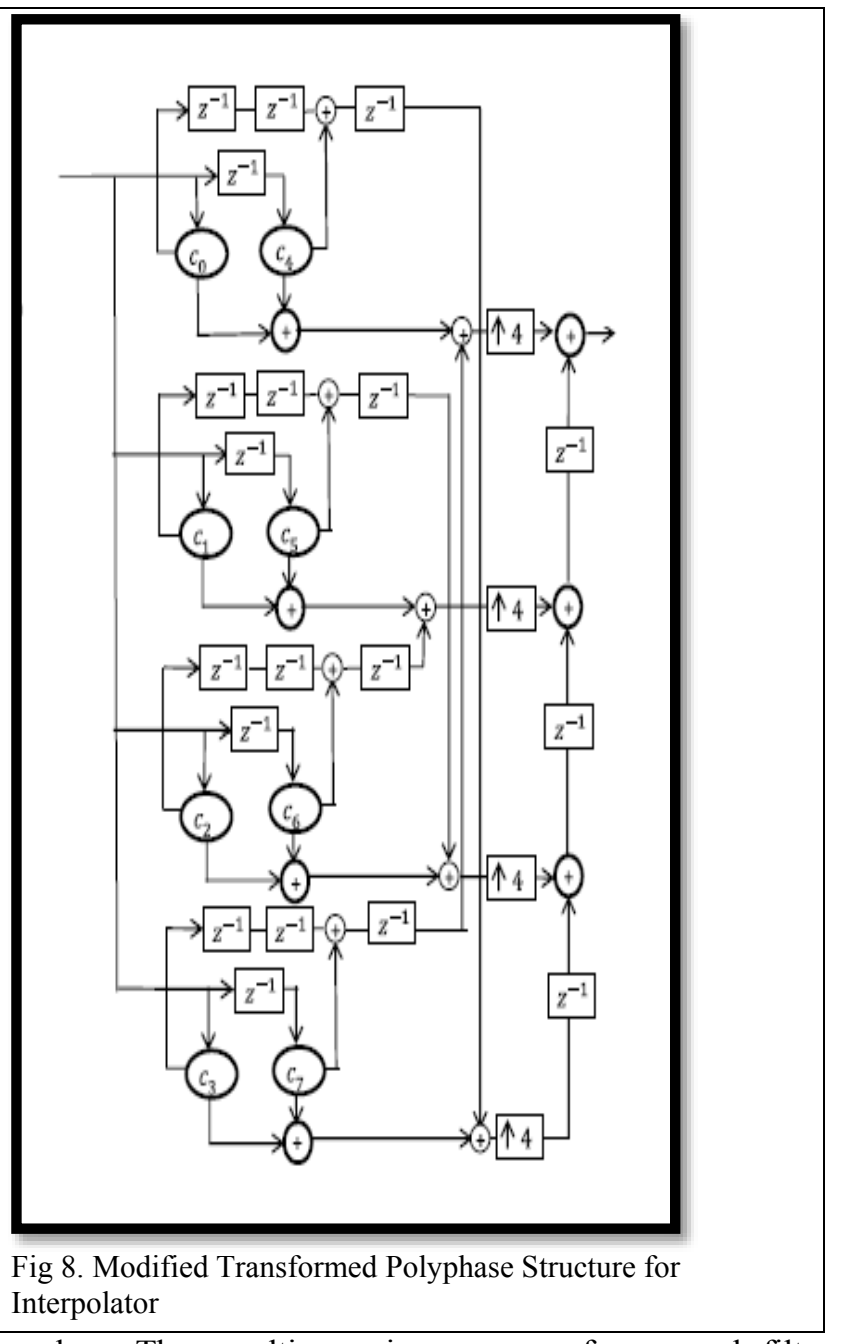

loss. The resulting gain response of proposed filter was given as Figure. 8.

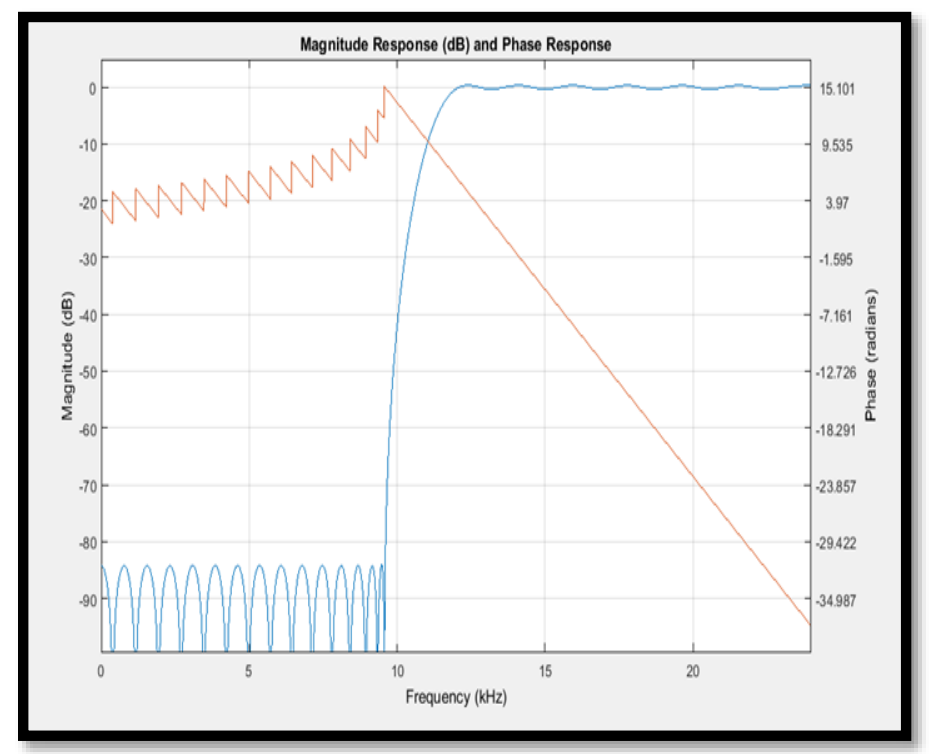

Fig 9 .Magnitude and Phase Response 
We have also provided the features of the proposed filter phase and magnitude, as given in Figures. 9 and 10, respectively.

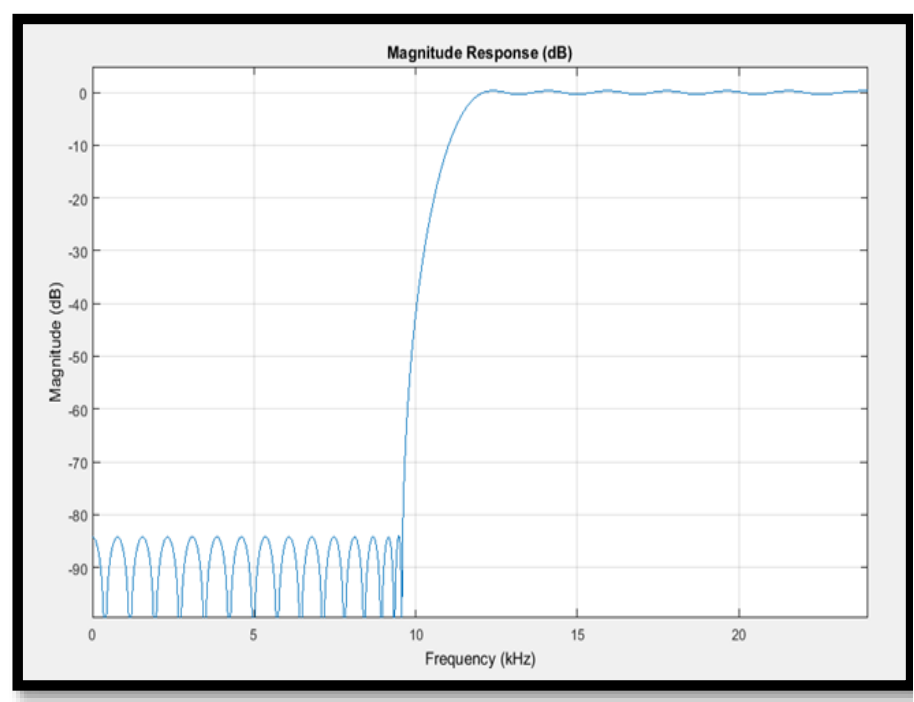

Fig 10 Magnitude Response

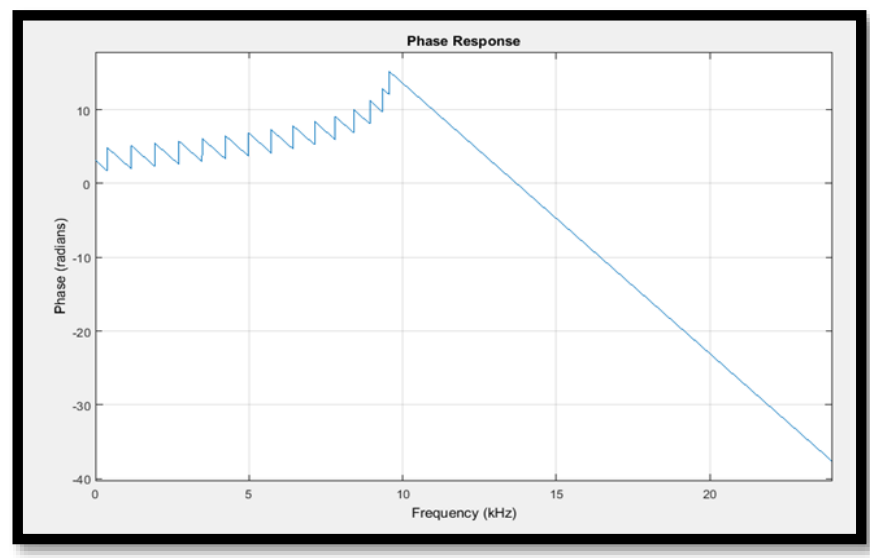

Fig 11. Phase Response

As they flow through a filter, all the frequency components of a signal are postponed. The time delay of the signal through the system under test as a function of frequency is also called a Group delay in a filter. The group delay for every subfilter was constant, which contributes to the linear process of the filter being presented and is expressed in Figure.11

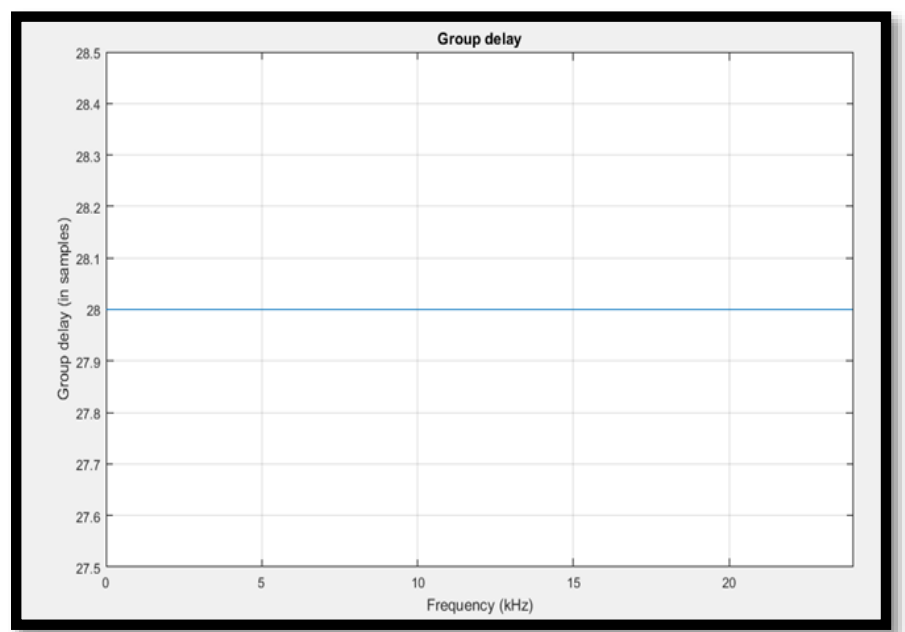

Fig 12.Group Delay

Many multipliers needed for presented filter was tabulated and compared with the techniques mentioned in $[24,25]$ in Table 8 .

Table.1 Hardware Complexity Comparision

\begin{tabular}{|lll|}
\hline S.No & Method & $\begin{array}{l}\text { Number } \\
\text { Multipliers }\end{array}$ \\
\hline $\mathbf{1}$ & Non-Uniform filter & 918 \\
\hline $\mathbf{2}$ & $\begin{array}{l}\text { VBW } \\
\text { method masking }\end{array}$ & 616 \\
\hline $\mathbf{3}$ & Proposed Filter & 57 \\
\hline
\end{tabular}

The number of multipliers is decreased by utilizing the coefficient symmetry of polyphase components. Whereas the multiplier requirement is almost minimized by manipulating the coefficient symmetry, which could easily be found in Table 1, the traditional polyphase framework empowers multiplier units to be similar to the filter order. Although, as coefficient symmetry is manipulated, the multiplier criteria decrease. Our proposed Table 1 solution reduces rm. The power consumption is effectively controlled by promoting the proposed filter in terms of complexity and delay.

Besides, this proposed filter explores whether the wideband channel is assisted. Regarding communication standards includes QPSK, FSK, and PSK are provided the input of the proposed filter. Then, the normalized magnitude-based frequency response is evaluated. The communication standards are realized using a proposed filter in Figs. 12. 


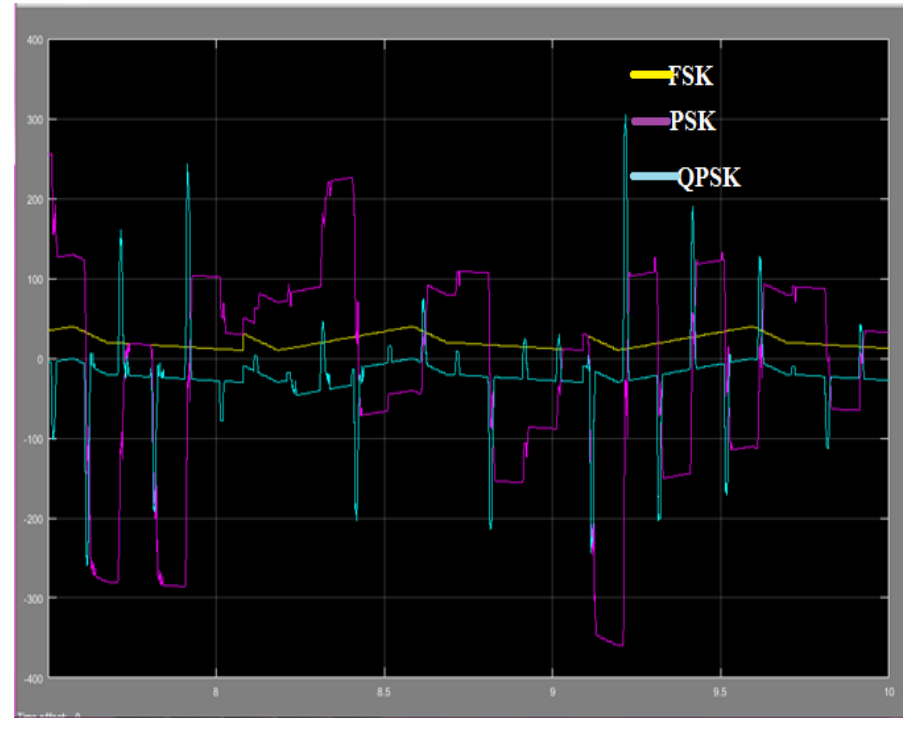

Fig 13. Communication Standard realized using proposed filter

\section{CONCLUSION}

The paper proposes the polyphase decomposition and coefficient symmetry incorporated into the Non-Maximally Coefficient Symmetry Multirate Filter Bank, recommended to the high-resolution wideband channel. In this proposal, a reconfigurable NMDFB architecture modifies the basic FRM structure. Moreover, polyphase decomposition and coefficient symmetry in the proposed filter is exploited in the proposed schemes. We have derived transfer function expressions under coefficient symmetry for polyphase FIR filters via the interpolator design transposed by the decimator design. In addition, we have provided the proposed filter bank's phase and magnitude properties. To improve the hardware efficiency and functionality of the proposed schemes, hardware-level optimization can also be implemented into the modified FRM techniques.

\section{REFERENCE}

[1] D'Arco, Mauro, Ettore Napoli, and Efstratios Zacharelos. "Digital Circuit for Seamless Resampling ADC Output Streams." Sensors 20.6 (2020): 1619.https://doi.org/10.3390/s20061619

[2] Owojori, Adedotun O., Temidayo O. Otunniyi, and Erastus O. Ogunti. "Digital Front-End for Software Defined Radio Wideband Channelizer." Communications on applied electronics 1.6 (2015): 25-35.

[3] Agiwal, Mamta, Abhishek Roy, and Navrati Saxena. "Next generation 5G wireless networks: A comprehensive survey." IEEE Communications Surveys \& Tutorials $18.3 \quad$ (2016): 16171655.DOI: $\underline{10.1109 / C O M S T .2016 .2532458}$

[4] Mahesh, R., et al. "Filter bank channelizers for multi-standard software defined radio receivers."
Journal of signal processing systems 62.2 (2011): 157-171.https://doi.org/10.1007/s11265-008-0327-y

[5] Mitola, J. (2000). Software radio architecture, John Wiley

[6] Hentschel, T., Henker, M., \& Fettweis, G. (1999). "The digital front-end of software radio terminals," IEEE Person. Commun. Magazine, pp. 40-46.

[7] Tato, Anxo. "Software defined radio: a brief introduction." Multidisciplinary Digital Publishing $\begin{array}{llll}\text { Institute Proceedings } 2.18 & \text { (2018): } 1196 .\end{array}$ https://doi.org/10.3390/proceedings2181196

[8] Rathinakumar, Saravana Manickam, Bozidar Radunovic, and Mahesh K. Marina. "ShiftFFT: An efficient approach to mitigate adjacent channel interference in OFDM systems." Proceedings of the 2nd International Workshop on Hot Topics in Wireless. 2015. https://doi.org/10.1145/2799650.2799658

[9] Kalathil, Shaeen, Bijili Sravan Kumar, and Elizabeth Elias. "Efficient design of multiplier-less digital channelizers using recombination non-uniform filter banks." Journal of King Saud UniversityEngineering $\quad$ Sciences $30.1 \quad$ (2018): 3137.https://doi.org/10.1016/j.jksues.2015.11.002

[10] Kalathil, Shaeen, and Elizabeth Elias. "Efficient design of non-uniform cosine modulated filter banks for digital hearing aids." AEU-International Journal of Electronics and Communications 69.9 (2015): 1314-

1320.https://doi.org/10.1016/j.aeue.2015.05.015

[11] Sapio, Adrian E., Marilyn Wolf, and Shuvra S. Bhattacharyya. "Compact modeling and management of reconfiguration in digital channelizer implementation." 2016 IEEE Global Conference on Signal and Information Processing (GlobalSIP). IEEE, 2016.DOI: $\underline{10.1109 / \text { GlobalSIP.2016.7905911 }}$

[12] Mahesh, R., et al. "Filter bank channelizers for multi-standard software defined radio receivers." Journal of signal processing systems 62.2 (2011): 157-171.https://doi.org/10.1007/s11265-008-0327-y

[13]CG, Mrs Kirti S. Vaidya Dr, Dr S. Dethe, and G. Akojwar. "Survey of Channelization Techniques For Digital Front End of Software Defined Radio." signal 6.5 (2017).

[14]Blok, Marek. "Sample rate conversion based on frequency response masking filter." 2018 International Conference on Signals and Electronic Systems (ICSES). IEEE, 2018.DOI: $10.1109 /$ ICSES.2018.8507320 
[15]Zhang, Wenxu, et al. "Low-complexity channelizer based on FRM for passive radar multi-channel wideband receiver." Circuits, Systems, and Signal Processing $\quad 39.1 \quad$ (2020): 420438.https://doi.org/10.1007/s00034-019-01192-0

[16]F. J. Harris, E. Venosa, X. Chen. and C. Dick, "Cascade non-maximally decimated filter banks form efficient variable bandwidth filters for wideband digital transceivers", In Digital Signal $\begin{array}{llll}\text { Processing } & (D S P), & 2017 & 22^{\text {nd }}\end{array}$ DOI: $\underline{\text { 10.1109/ICDSP.2017.8096064 }}$

[17] International Conference on, pp. 1-5, 2017 [17] Mahesh, R., and A. Prasad Vinod. "Reconfigurable frequency response masking filters for software radio channelization." IEEE Transactions on Circuits and Systems II: Express Briefs 55.3 (2008): 274-278.DOI: 10.1109/ICETIETR.2018.8529072

[18] Lim, Yong. "Frequency-response masking approach for the synthesis of sharp linear phase digital filters." IEEE transactions on circuits and systems 33.4 (1986): 357-364.DOI: $10.1109 /$ TCS.1986.1085930

[19] Ramya, R., and S. Moorthi. "Frequency response masking based FIR filter using approximate multiplier for bio-medical applications." Sādhanā 44.11 (2019): 225.https://doi.org/10.1007/s12046019-1186-x

[20]Zhou, Daniel. A review of polyphase filter banks and their application. No. AFRL-IF-RS-TR-2006277. AIR FORCE RESEARCH LAB ROME NY INFORMATION DIRECTORATE, 2006.

[21] Harris, Fred, et al. "Wideband 160-channel polyphase filter bank cable TV channeliser." IET Signal processing $5.3 \quad$ (2011): 325 332.DOI: $\underline{10.1049 / \text { iet-spr.2010.0031 }}$

[22] Wang, Luyun, Ronggang Qi, and Guoan Bi. "Structure of non-maximally decimated filter bank derived by MSFGs." Electronics Letters 53.16 (2017):

$1106-$ 1107.https://doi.org/10.1049/el.2017.0744

[23]Chandra, Abhijit, and Sudipta Chattopadhyay. "Design of hardware efficient FIR filter: A review of the state-of-the-art approaches." Engineering Science and Technology, an International Journal $19.1 \quad$ (2016): 212226.https://doi.org/10.1016/j.jestch.2015.06.006

[24] V. Sakthivel, E. Elias, Low complexity reconfigurable channelizers using non-uniform filter banks. Comput. Electr. Eng. 68, 389-403 (2018). https://doi.org/10.1016/j.compeleceng.2018.04.015

[25]N. Haridas, E. Elias, Reconfigurable farrow structure-based frm filters for wireless communication systems. Circuits Syst. Signal Process. 36(1), 315-338 (2017). https://doi.org/10.1007/s00034-016-0309-4

[26] PP Vaidyanathan, Multirate Systems and Filter Banks (Prentice-Hall, Englewood Cliffs, NJ, 2006)

[27] R. Mahesh, A.P. Vinod, Reconfigurable frequency response masking filters for software radio channelization. IEEE Trans. Circuits Syst. II Express Briefs 55(3), 274-278 (2008). DOI: 10.1109/TCSII.2008.918996

[28] Sudharman, Sudhi, and T. S. Bindiya. "Design of Reconfigurable FRM Channelizer using Resource Shared Non-maximally Decimated Masking Filters." Journal of Signal Processing Systems (2020): 110.https://doi.org/10.1007/s11265-020-01615-1

[29] Yu, Ya Jun, Dong Shi, and Robert Bregovic. "On the complexity reduction of polyphase linear phase FIR filters with symmetric coefficient implementation." 2009 IEEE International Symposium on Circuits and Systems. IEEE, 2009.Doi: 10.1109/ISCAS.2009.5117739

[30]P.P. Vaidyanathan, Multirate Systems and Filter Banks (Prentice-Hall, Englewood Cliffs, NJ, 2006)

\section{Creative Commons Attribution License 4.0 (Attribution 4.0 International, CC BY 4.0)}

This article is published under the terms of the Creative Commons Attribution License 4.0 https://creativecommons.org/licenses/by/4.0/deed.en_US 